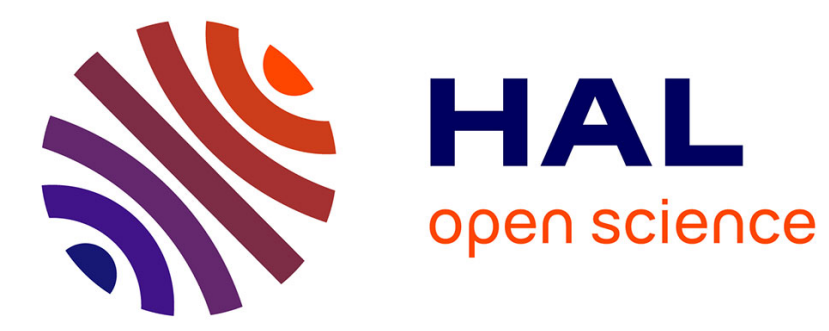

\title{
Dispersive properties of linear chains of lossy metal nanoparticles
}

\author{
Matteo Conforti, Massimiliano Guasoni
}

\section{To cite this version:}

Matteo Conforti, Massimiliano Guasoni. Dispersive properties of linear chains of lossy metal nanoparticles. Journal of the Optical Society of America B, 2010, 27 (8), pp.1576-1582. 10.1364/JOSAB.27.001576 . hal-02397510

\section{HAL Id: hal-02397510 https://hal.science/hal-02397510}

Submitted on 6 Dec 2019

HAL is a multi-disciplinary open access archive for the deposit and dissemination of scientific research documents, whether they are published or not. The documents may come from teaching and research institutions in France or abroad, or from public or private research centers.
L'archive ouverte pluridisciplinaire HAL, est destinée au dépôt et à la diffusion de documents scientifiques de niveau recherche, publiés ou non, émanant des établissements d'enseignement et de recherche français ou étrangers, des laboratoires publics ou privés. 


\title{
Dispersive properties of linear chains of lossy
}

\section{metal nanoparticles}

\author{
Matteo Conforti and Massimiliano Guasoni \\ CNISM and Dipartimento di Ingegeria dell'Informazione, Università di Brescia, \\ Via Branze 38, 25123 Brescia, Italy \\ corresponding author: matteo.conforti@ing.unibs.it
}

We study the propagation characteristics of optical signals in waveguides composed of linear periodic arrangements of metallic nanoparticles embedded in a dielectric host. We find the complex Bloch band diagram for the guided modes including material losses by employing Mie scattering theory as well as coupled dipole approximations. The results of the model are validated through finite element solution of Maxwell's equations.

(c) 2010 Optical Society of America

OCIS codes: $260.3910,130.2790,240.6680,260.2030,000.4430$

\section{Introduction}

Since the pioneering paper by Quinten et al. [1] the subject of light propagation in linear chains of metal nanoparticles has attracted a lot of research efforts [2-12]. This 
interest is mainly motivated by the wide range of potential applications that sweep from the realization of biologic nanosensors [13], to sub-wavelength imaging [14], to the merging of electronic circuits to photonic devices [15].

All the relevant properties of a guiding structure are embedded in the dispersion relation $k=k(\omega)$, i.e. the functional relation between the wavevector of the propagating mode and the operating frequency. Several methods to calculate the dispersion relation for linear chains of nanoparticles were based on the coupled-dipole approximation (CDA), where the electromagnetic field is supposed to be the sum of the field emitted by the spheres, treated as point dipoles with a certain polarizability $\alpha(\omega)$. The first studies considered only nearest neighbor interaction and neglected retardation effects, i.e. considered the static polarizability of the particles and only the near-field of the dipole $[2,3]$. Latter studies showed that retardation effects are fundamental and cannot be neglected in the computation of dispersion relation [4]. The inclusion of these aspects leads to a dispersion equation expressed as an infinite series, that diverges when the unavoidable losses of the the metal are taken into account. Analytic continuation techniques were employed to express the dipole sum in term of Polylogarithmic functions [8], whose properties were studied in details in Ref. [10]. In all these studies, however, the effect of losses was neglected or treated at the first order by perturbation techniques. The complex dispersion relation fully taking into account the losses in metal was solved by Koenderink et al. [9], by fixing a real wavevector and finding a complex frequency. This method is however unphysical 
when dealing with a waveguide, and gives bad results when the group velocity of the propagating mode becomes small or when the decay rate is considerable.

In this paper we derive the dispersion relation for nanoparticle chains by exploiting Mie scattering method. This dispersion relation is exact when all modes are considered. In a certain range of parameters only the first order spherical vector harmonics need to be considered, and simple expressions for longitudinal and transverse modes are obtained. Interestingly enough, we show that the dispersion relation reduces to the usual one obtained by coupled dipole approximation, provided that the polarizability of the spheres is calculated by Mie scattering coefficients.

Next we calculate the complex band diagram by numerically solving the dispersion relation for lossy particles, by fixing a real frequency and finding a complex wavevector. We find a complex dispersion relation that strongly differs from previous studies $[9,10]$ : losses modify even the real part of the propagation constant (an effect not captured by first order perturbation) and prevent the existence of resonator modes [7] characterized by vanishing group velocity.

To conclude we compare the results of the Mie model with the exact Bloch modes dispersion calculated by finite element solution of Maxwell's equations. We revisit the finite element method formulation for the calculation of three dimension periodic crystal bands. The method yields a quadratic eigenvalue equation in the Bloch wavevector modulus. In this case frequency is a parameter so that the strong dispersion of the metal is easily taken into account. 
The paper is organized as follows. In Sec. II we find the complex dispersion relation of linear chains of nanoparticles following the Mie scattering approach. In Sec. III we show the complex band diagram for a case of interest, highlighting the difference with previous results. In Sec. IV we develop the proper formulation of finite element and compare the result of finite element simulation and analytical approach. Section V contains the concluding remarks.

\section{Dispersion relation of nanoparticle chains: Mie theory approach}

In this section we develop a model to analyze the properties of a periodic linear chain of nanospheres based on the generalized Mie theory of Gerardy and Ausloos for a cluster of spheres [16]. The nanospheres have radius $R$, center-to-center spacing $d$, dielectric constant $\epsilon_{s}$, and are embedded in an infinite matrix with dielectric constant $\epsilon_{m}$. Mie theory states that any field can be expressed as linear combination of the vector spherical harmonics (VSH) of the first and third kind $\vec{m}_{l m}^{1}, \vec{m}_{l m}^{3}, \vec{n}_{l m}^{1}$ and $\vec{n}_{l m}^{3}$ because they constitute a complete and orthogonal set of vector basis functions [16-18]. Longitudinal waves described by functions $\vec{l}_{l m}$ are neglected, because we assume that the dielectric constants of the spheres and the matrix are isotropic.

We can expand any arbitrary incident electromagnetic field $\left(\vec{E}_{i}, \vec{H}_{i}\right)$ in the matrix as linear combination of vector spherical harmonics of the first kind $\vec{m}_{l m}^{1}(n)$ and $\vec{n}_{l m}^{1}(n)$ centered in the $n$-th sphere: 


$$
\begin{array}{r}
\vec{E}_{i}=\sum_{l m}\left\{a_{i, l m}(n) \vec{m}_{l m}^{1}(n)+b_{i, l m}(n) \vec{n}_{l m}^{1}(n)\right\} \\
\vec{H}_{i}=\frac{k_{M}}{i \mu_{0} \omega} \sum_{l m}\left\{b_{i, l m}(n) \vec{m}_{l m}^{1}(n)+a_{i, l m}(n) \vec{n}_{l m}^{1}(n)\right\}
\end{array}
$$

where $n$ refers to the $n$-th sphere in the chain. The index $l$ sweeps from 1 to infinity, while $m$ from $-l$ to $+l$, and $a_{i, l m}(n)$ and $b_{i, l m}(n)$ are the coefficients of the linear combination. $k_{M}=\omega \sqrt{\epsilon_{m}} / c$ is the wave-vector in the matrix, $\mu_{0}$ is the magnetic permeability of vacuum and $\omega$ is the angular pulsation of the input field.

Starting from here and for the rest of the paper we develop our calculation basing upon the electric field only, as it is usually done [16]. In the frame centered in the $n$-th sphere the total electric field $\vec{E}(n)$ can be expressed as the sum of the incident field [that is linear combination of VSH of first kind $\vec{m}_{l m}^{1}(n)$ and $\vec{n}_{l m}^{1}(n)$ ] and of the scattered field [that is linear combination of VSH of the third kind $\vec{m}_{l m}^{3}(n)$ and $\left.\vec{n}_{l m}^{3}(n)\right][16]:$

$$
\begin{array}{r}
\vec{E}(n)=\sum_{l m}\left\{a_{l m}(n) \vec{m}_{l m}^{1}(n)+b_{l m}(n) \vec{n}_{l m}^{1}(n)+\right. \\
\left.c_{l m}(n) \vec{m}_{l m}^{3}(n)+d_{l m}(n) \vec{n}_{l m}^{3}(n)\right\} .
\end{array}
$$

Functions $\vec{m}_{l m}^{1}(n)$ and $\vec{n}_{l m}^{1}(n)$ are excited both by the input field and by the scattered field of the other spheres, that is sum of functions of the third kind $\vec{m}_{l m}^{3}(v)$ and $\vec{n}_{l m}^{3}(v)$ with $v \neq n$. For this reason the coefficients $a_{l m}(n)$ and $b_{l m}(n)$ in Eq.(2) differ from $a_{i, l m}(n)$ and $b_{i, l m}(n)$ in Eq.(1), relative to the input field only. In general, any 
function $\vec{m}_{l m}^{1}(n)$ and $\vec{n}_{l m}^{1}(n)$ produces a scattered vector function that is, respectively, $\vec{m}_{l m}^{3}(n)$ and $\vec{n}_{l m}^{3}(n)$. Coefficients $a_{l m}(n), b_{l m}(n), c_{l m}(n)$ and $d_{l m}(n)$ are linked by means of the scattering coefficients of the Mie theory [16]:

$$
\begin{aligned}
& c_{l m}(n)=\Gamma_{l}(n) a_{l m}(n), \\
& d_{l m}(n)=\Delta_{l}(n) b_{l m}(n),
\end{aligned}
$$

where $\Gamma_{l}(n)$ and $\Delta_{l}(n)$ are the scattering coefficients related to the $n$-th sphere. In the case described in this paper, all the spheres have the same radius and dielectric constant so that the scattering coefficients are equal for all the spheres and do not depend on $n$. In this case generalized scattering coefficients $\Delta_{m}$ and $\Gamma_{n}$ reduces to the scattering coefficients of the single isolated sphere (usually called $a_{m}$ and $b_{m}$ in literature [18], that differ from $a_{m}$ and $b_{m}$ in our notation).

The coefficients $a_{l m}(n)$ are the sum of $a_{i, l m}(n)$ due to the input field and of all the contributions $T_{p q l m}(v, n) c_{p q}(v)$ and $C_{p q l m}(v, n) d_{p q}(v)$ due respectively to the scattered functions $\vec{m}_{p q}^{3}(v)$ and $\vec{n}_{p q}^{3}(v)$ of all the other spheres in the matrix, so that:

$$
\begin{array}{r}
a_{l m}(n)=c_{l m}(n) / \Gamma_{l}=a_{i, l m}(n)+ \\
\sum_{v \neq n} \sum_{p q}\left\{T_{p q l m}(v, n) c_{p q}(v)+C_{p q l m}(v, n) d_{p q}(v)\right\} .
\end{array}
$$

$T_{p q l m}(v, n)$ is the coupling coefficient between $\vec{m}_{l m}^{1}(n)$ and $\vec{m}_{p q}^{3}(v)$ in the frame centered in the $n$-th sphere, or, equivalently, the coupling coefficient between $\vec{n}_{l m}^{1}(n)$ 
and $\vec{n}_{p q}^{3}(v) . C_{p q l m}(v, n)$ is the coupling coefficient between $\vec{n}_{l m}^{1}(n)$ and $\vec{m}_{p q}^{3}(v)$ in the frame centered in the $n$-th sphere, or, equivalently, the coupling coefficient between $\vec{m}_{l m}^{1}(n)$ and $\vec{n}_{p q}^{3}(v)$. Similar arguments hold true for coefficients $b_{l m}(n)$, so that it is possible to write:

$$
\begin{array}{r}
b_{l m}(n)=d_{l m}(n) / \Delta_{l}=b_{i, l m}(n)+ \\
\sum_{v \neq n} \sum_{p q}\left\{C_{p q l m}(v, n) c_{p q}(v)+T_{p q l m}(v, n) d_{p q}(v)\right\} .
\end{array}
$$

Equations (4-5) constitute a system that allows to calculate the coefficients $c_{p q}(n)$ and $d_{p q}(n)$ from the knowledge of the input field (coefficients $a_{i, p q}(n)$ and $\left.b_{i, p q}(n)\right)$. By inserting $c_{p q}(n)$ and $d_{p q}(n)$ in Eqs. $(2,3)$ the total field in the matrix can be exactly calculated.

It is often possible to simplify the system $(4,5)$ by considering only the scattering coefficients that are significantly different from 0 . A drastic simplification of the treatment is possible when only the first coefficient $\Delta_{1}$ is significant: this usually happens when the radius $R$ is sufficiently smaller than the wavelength of the input field. For the rest of the paper we actually work under this assumption. In this way only functions $\vec{n}_{1-1}^{1}, \vec{n}_{10}^{1}$ and $\vec{n}_{11}^{1}$ are interacting. In fact by setting $\Gamma_{l}=0$ and $\Delta_{l+1}=0$ for any $l \geq 1$, the coefficients $c_{p q}$ and $d_{p q}$ in Eq.(4) and Eq.(5) are equal to 0, except $d_{1-1}$, $d_{10}$ and $d_{11}$, whose values are now given by Eq.(5) with $m=-1,0,1$ : 


$$
b_{i, 1 m}(n)=d_{1 m}(n) / \Delta_{1}-\sum_{v \neq n} \sum_{q=-1}^{1} T_{1 q 1 m}(v, n) d_{1 q}(v) .
$$

If the chain of nanospheres is located on the $x-y$ plane, then the functions $\vec{n}_{10}^{1}$ are decoupled from $\vec{n}_{1-1}^{1}$ and $\vec{n}_{11}^{1}$, because it can be shown (for example using results in [19]) that $T_{101-1}(v, n)=T_{1011}(v, n)=T_{1-110}(v, n)=T_{1110}(v, n)=0$. Moreover, being the spheres equidistant, $T_{1010}(v, n)=T_{1010}(v-n)$; of course also for the other coupling coefficients $(n, v)$ can be substituted by $(v-n)$. From Eq.(6) it is then possible to write, for the coefficients $d_{10}$ :

$$
\begin{array}{r}
b_{i, 10}(n)=\Delta_{1}^{-1} d_{10}(n)-\sum_{v \neq n} T_{1010}(v-n) d_{10}(v) \\
=U_{1010}(n) * d_{10}(n)
\end{array}
$$

where the symbol $*$ denotes the discrete convolution and we put $U_{1010}(0)=\Delta_{1}^{-1}$ and $U_{1010}(n)=-T_{1010}(n)$ for $n \neq 0$. Reasoning in a similar way, from Eq.(6) we can obtain for the coefficients $d_{1-1}$ and $d_{11}$ :

$$
\begin{gathered}
b_{i, 1-1}(n)=U_{1-11-1}(n) * d_{1-1}(n)+U_{111-1}(n) * d_{11}(n) \\
b_{i, 11}(n)=U_{1-111}(n) * d_{1-1}(n)+U_{1111}(n) * d_{11}(n)
\end{gathered}
$$

where $U_{1-11-1}(0)=U_{1111}(0)=\Delta_{1}^{-1}, U_{111-1}(0)=U_{1-111}(0)=0$, and for any $n \neq 0$ 
$U_{1-11-1}(n)=-T_{1-11-1}(n), U_{111-1}(n)=-T_{111-1}(n), U_{1-111}(n)=-T_{1-111}(n)$ and $U_{1111}(n)=-T_{1111}(n)$. Moreover, from [19] it is possible to see that $T_{1-11-1}(n)=$ $T_{1111}(n)$ and $T_{111-1}(n)=T_{1-111}(n)$, so that $U_{1-11-1}(n)=U_{1111}(n)$ and $U_{111-1}(n)=$ $U_{1-111}(n)$

As far as the calculation of the coupling coefficients, a lot of efforts have been done in order to calculate them in a straightforward and fast way; in this sense, an excellent result has been reached in Ref. [19], where we can find simple analytical formulas. Following the treatment it is possible to show that for $n \neq 0$ :

$$
\begin{aligned}
& T_{1010}(n)=-i \frac{3}{2} \frac{e^{i k_{M}|d \cdot n|}}{k_{M}|d \cdot n|}+\frac{3}{2} \frac{e^{i k_{M}|d \cdot n|}}{\left(k_{M}|d \cdot n|\right)^{2}}+i \frac{3}{2} \frac{e^{i k_{M}|d \cdot n|}}{\left(k_{M}|d \cdot n|\right)^{3}}, \\
& T_{1-111}(n)=i \frac{3}{4} \frac{e^{i k_{M}|d \cdot n|}}{k_{M}|d \cdot n|}-\frac{9}{4} \frac{e^{i k_{M}|d \cdot n|}}{\left(k_{M}|d \cdot n|\right)^{2}}-i \frac{9}{4} \frac{e^{i k_{M}|d \cdot n|}}{\left(k_{M}|d \cdot n|\right)^{3}}, \\
& T_{1111}(n)=-i \frac{3}{4} \frac{e^{i k_{M}|d \cdot n|}}{k_{M}|d \cdot n|}-\frac{3}{4} \frac{e^{i k_{M}|d \cdot n|}}{\left(k_{M}|d \cdot n|\right)^{2}}-i \frac{3}{4} \frac{e^{i k_{M}|d \cdot n|}}{\left(k_{M}|d \cdot n|\right)^{3}} .
\end{aligned}
$$

Eq.(7) can be rewritten in the "spatial frequency" (or wavevector) domain by using the Discrete Time Fourier Transform, so that:

$$
\hat{b}_{i, 10}(k)=\hat{U}_{1010}(k) \hat{d}_{10}(k)
$$

where $\hat{b}_{i, 10}(k), \hat{U}_{1010}(k)$ and $\hat{d}_{10}(k)$ are respectively the Fourier transforms of the sequences $b_{i, 10}(n), U_{1010}(n)$ and $d_{10}(n)$. It is then straightforward to calculate $d_{10}(n)$ as a linear filtering of the input coefficients $b_{i, 10}(n)$ by means of the transfer function $1 / \hat{U}_{1010}(k)$ 
We can check for the existence of a self-sustaining mode of the chain by forcing a vanishing input field (i.e. $b_{i, 10}(n)=0$ ) and by looking for those $k$ for which $d_{10}(n) \neq 0$, that is to find those $k$ that make $\hat{U}_{1010}(k)=0$.

Also Eq.(8) can be rewritten in the frequency domain:

$$
\begin{array}{r}
\hat{b}_{i, 1-1}(k)=\hat{U}_{1111}(k) \hat{d}_{1-1}(k)+\hat{U}_{1-111}(k) \hat{d}_{11}(k) \\
\hat{b}_{i, 11}(k)=\hat{U}_{1-111}(k) \hat{d}_{1-1}(k)+\hat{U}_{1111}(n) \hat{d}_{11}(k)
\end{array}
$$

where the relations $\hat{U}_{1-11-1}(k)=\hat{U}_{1111}(k)$ and $\hat{U}_{1-111}(k)=\hat{U}_{111-1}(k)$ are used. Once again, the coefficients $d_{1-1}(n)$ and $d_{11}(n)$ are easily calculable as a linear filtering of the input coefficients $b_{i, 1-1}(n)$ and $b_{i, 11}(n)$. As before we force the input coefficients $b_{i, 1-1}(n)$ and $b_{i, 11}(n)$ to zero in order to find the modes of the chain. In this case the system $(11)$ has nontrivial solutions $\hat{d}_{1-1}(k)$ and $\hat{d}_{11}(k)$ only if $\hat{U}_{1111}(k) \pm \hat{U}_{111-1}(k)=0$, that correspond to $\hat{d}_{11}(k)= \pm \hat{d}_{1-1}(k)$, i.e. $d_{11}(n)= \pm d_{1-1}(n)$.

We look for $k$ that solves $\hat{U}_{1010}(k)=0$ in order to find the first mode of the chain; being $U_{1010}(0)=\Delta_{1}^{-1}$ and using Eq.(9) for $T_{1010}(n)$, we can write:

$$
\hat{U}_{1010}(k)=\Delta_{1}^{-1}-\sum_{n=1}^{\infty} T_{1010}(n) e^{-i k n}-\sum_{n=1}^{\infty} T_{1010}(-n) e^{+i k n}=0
$$

that is (from the definition of polylogarithm [20]): 


$$
\begin{aligned}
0=\Delta_{1}^{-1} & +i \frac{3}{2} \frac{\operatorname{Li}_{1}\left(e^{i\left(k_{M} d-k\right)}\right)+\operatorname{Li}_{1}\left(e^{i\left(k_{M} d+k\right)}\right)}{k_{M} d} \\
& -\frac{3}{2} \frac{\operatorname{Li}_{2}\left(e^{i\left(k_{M} d-k\right)}\right)+\operatorname{Li}_{2}\left(e^{i\left(k_{M} d+k\right)}\right)}{\left(k_{M} d\right)^{2}} \\
& -i \frac{3}{2} \frac{\operatorname{Li}_{3}\left(e^{i\left(k_{M} d-k\right)}\right)+\operatorname{Li}_{3}\left(e^{i\left(k_{M} d+k\right)}\right)}{\left(k_{M} d\right)^{3}},
\end{aligned}
$$

where $\operatorname{Li}_{p}(x)$ is the polylogarithm function of order $p . \hat{U}_{1010}(k)$ is symmetric and periodic of $\pi$ in $k$, so that we can limit the search of the real part of $k$ between 0 and $\pi$, that is coherent with the fact that the system is periodic. If $k$ is the solution of Eq.(13), then the propagation constant of the mode is $\beta=k / d$ and $d_{10}(n)=e^{i k n}$, so that the electric and magnetic field of the mode are:

$$
\begin{array}{r}
\vec{E}=\sum_{n=-\infty}^{\infty} \vec{n}_{10}^{3}(n) e^{i k n}, \\
\vec{H}=\frac{k_{M}}{i \mu_{0} \omega} \sum_{n=-\infty}^{\infty} \vec{m}_{10}^{3}(n) e^{i k n} .
\end{array}
$$

Along the direction of alignment ( $x$ axis) functions $\vec{n}_{10}^{3}$ posses only $z$ component, making the electric field transverse with respect to the direction of propagation.

The second and third mode of the chain are found by solving $\hat{U}_{1111}(k) \pm \hat{U}_{1-111}(k)=$ 0. Being $U_{1111}(0)=\Delta_{1}^{-1}, U_{1-111}(0)=0$ and using Eq.(9) for $T_{1111}(n)$ and $T_{1-111}(n)$, it is possible to recast the second mode equation in this way: 


$$
\hat{U}_{1111}(k)+\hat{U}_{1-111}(k)=0
$$

that is

$$
\begin{array}{r}
0=\Delta_{1}^{-1}+3 \frac{\operatorname{Li}_{2}\left(e^{i\left(k_{M} d-k\right)}\right)+\operatorname{Li}_{2}\left(e^{i\left(k_{M} d+k\right)}\right)}{\left(k_{M} d\right)^{2}} \\
+i 3 \frac{\operatorname{Li}_{3}\left(e^{i\left(k_{M} d-k\right)}\right)+\operatorname{Li}_{3}\left(e^{i\left(k_{M} d+k\right)}\right)}{\left(k_{M} d\right)^{3}} .
\end{array}
$$

The solution of Eq.(16) $(0<R e(k)<\pi)$ imposes $\hat{d}_{1-1}(k)=\hat{d}_{11}(k)$, then $d_{1-1}(n)=$ $d_{11}(n)$, so that the electric and magnetic field of the mode are:

$$
\begin{array}{r}
\vec{E}=\sum_{n=-\infty}^{\infty}\left(\vec{n}_{1-1}^{3}(n)+\vec{n}_{11}^{3}(n)\right) e^{i k n}, \\
\vec{H}=\frac{k_{M}}{i \mu_{0} \omega} \sum_{n=-\infty}^{\infty}\left(\vec{m}_{1-1}^{3}(n)+\vec{m}_{11}^{3}(n)\right) e^{i k n} .
\end{array}
$$

Along the direction of alignment ( $x$ axis) functions $\vec{n}_{10}^{3}$ posses only $x$ component, making the electric field longitudinal with respect to the direction of propagation.

The equation for the third mode is $\hat{U}_{1111}(k)-\hat{U}_{1-111}(k)=0$, and has the same formulation of Eq.(13): the first and third mode are degenerate. This fact is not surprising since the third mode is simply a ninety-degree rotation of the first one around the axis along which the chain is lined up. This makes the two modes degenerate because of the cylindrical rotational symmetry of the system. 
Eq.(13) and Eq.(16) have to be solved in the complex plane. If both spheres and the matrix have purely real dielectric constant, then the system is lossless and it can be shown that $\left|\Delta_{1}\right|^{2}=-\operatorname{Re}\left(\Delta_{1}\right)$ : this implies that $\Delta_{1}^{-1}=\Delta_{1}^{*} /\left|\Delta_{1}\right|^{2}=$ $-1-i \operatorname{Im}\left(\Delta_{1}\right) / \operatorname{Re}\left(\Delta_{1}\right)$, so that $\operatorname{Re}\left(\Delta_{1}^{-1}\right)=-1$. It can be also shown that $\operatorname{Re}\left(\hat{U}_{1010}(k)-\right.$ $\left.\Delta_{1}^{-1}\right)=\operatorname{Re}\left(\hat{U}_{1111}(k)+\hat{U}_{1-111}(k)-\Delta_{1}^{-1}\right)=1$ for any $k$ real with $k_{M} d<k<\pi$. As a consequence, under this last condition $\operatorname{Re}\left(\hat{U}_{1010}(k)\right)=\operatorname{Re}\left(\hat{U}_{1111}(k)+\hat{U}_{1-111}(k)\right)=0$ when the system is lossless. It is then possible to look for real $k$ solutions between $k_{M} d$ and $\pi$ (that is, under the ligth line) by solving $\operatorname{Im}\left(\hat{U}_{1010}(k)\right)=0$ and $\operatorname{Im}\left(\hat{U}_{1111}(k)+\hat{U}_{1-111}(k)\right)=0$. The correspondent modes propagate without damping.

When the system is lossy, the condition $\operatorname{Re}\left(\hat{U}_{1010}(k)-\Delta_{1}^{-1}\right)=\operatorname{Re}\left(\hat{U}_{1111}(k)+\hat{U}_{1-111}(k)-\right.$ $\left.\Delta_{1}^{-1}\right)=1$ remain verified $\left(k_{M} d<k<\pi\right)$, but $\operatorname{Re}\left(\Delta_{1}^{-1}\right) \neq-1$ so there does not exist a real $k$ that solves $\operatorname{Re}\left(\hat{U}_{1010}(k)\right)=0$ or $\operatorname{Re}\left(\hat{U}_{1111}(k)+\hat{U}_{1-111}(k)\right)=0$ : solutions $k$ have to be found in the complex plane, as expected.

Equations (13) and (16) can be related to the dispersion relation for the transverse and longitudinal modes obtained by CDA $[4,9]$. In fact (13) and (16) can be rewritten as

$$
\begin{aligned}
1+\frac{\alpha(\omega)}{4 \pi d^{3} \epsilon_{m} \epsilon_{0}} \Sigma_{T}(\omega, \beta) & =0 \\
1-2 \frac{\alpha(\omega)}{4 \pi d^{3} \epsilon_{m} \epsilon_{0}} \Sigma_{L}(\omega, \beta) & =0
\end{aligned}
$$

where $\beta=k / d$ is the wavevector of the mode, $\alpha(\omega)=i 3 c^{3} \Delta_{1}(\omega) 4 \pi \epsilon_{m} \epsilon_{0} /\left({\sqrt{\epsilon_{M}}}^{3} \omega^{3}\right)$ is the "exact" polarizability of the spheres calculated by Mie scattering coefficients [21], 
and $\Sigma_{L}(\omega, \beta)$ and $\Sigma_{T}(\omega, \beta)$ are sum of polylogarithmic functions and depend on $\omega$ through $k_{M}(\omega)$.

This correspondence tells us that i) CDA is exact when the polarizability of spheres is calculated from Mie scattering coefficients and the particle radius (separation) are small (large) with respect to wavelength in the matrix; ii) we can easily obtain more accurate dispersion relation for bigger particles by including more vector sperical harmonics in $(4,5)$.

We want to underline that obtaining the coupled dipole approximation from the exact Mie theory shows exactly what approximations have been done, and indicates the way to improve the accuracy of the treatment when required. In fact in previous works the CDA was obtained by simply assuming that the spheres behave as point dipoles with a certain polarizability, and the mathematical formulation of the scattered fields is simply assumed. By means of Mie theory we were able to calculate the field scattered by the nanoparticles and the dispersion relation to a degree of accuracy that is fixed by the number of the considered vector spherical harmonics. In the following numerical examples we will show that the lowest order approximation furnish good enough results for small spheres, with size comparable to that usually exploited in real experiments. 


\section{Complex dispersion relation}

In this section we solve Eqs.(18-19) for a case of interest, by considering silver nanoparticles embedded in a glass substrate. In contrast to previous studies we solve (18-19) for a complex propagation constant $\beta$ at a fixed real value of frequency $\omega$. This is the natural way to proceed when studying a waveguide: in fact in the physical situation an electromagnetic field at a fixed real frequency impinges on the waveguide and excites some guided modes that propagates with a possibly complex propagation constant. The imaginary part of $\beta$ fixes the decay length of the guided modes along the structure. The dual way to proceed (fixing a real $\beta$ and find a complex $\omega$ ) was used un previous studies [9] to circumvent some mathematical problems. However this way to proceed is suitable for chains of finite length (i.e. resonators), where we expect to excite a vibrating mode along all the chain, characterized by a real $\beta$, and to see its decay in time fixed by imaginary part of frequency. When the imaginary part of $\beta$ and $\omega$ are small, and away from zero group velocity points, the two methods give approximately the same results. However in proximity of vanishing group velocity or in presence of strong losses (as in the case of metals at optical frequencies) the two methods give totally different results, as we shall see in this section.

We consider particle with radius $R=25 \mathrm{~nm}$ with a center to center spacing $d=$ $75 \mathrm{~nm}$ and we take the dielectric constant of glass $\epsilon_{m}=n_{m}^{2}=2.25$. For what concerns the metal, we used a fitting model based on the dielectric constant of silver tabulated 
in [22]. We decided not to use the popular Drude model, since it is not very accurate in the visible range, especially for what concerns absorption.

Figure 1 (Fig. 2) shows the frequency of the guided modes as a function of the real (imaginary) part of the propagation constant. Let's start our discussion by considering first dispersion curves calculated neglecting losses (dotted curves): we have one longitudinal mode $(L)$ and two transverse modes $\left(T_{1}\right.$ and $\left.T_{2}\right)$. Modes $L$ and $T_{1}$ are the usual longitudinal and transverse modes discussed previously in literature [4] and have purely real propagation constant under the light line $\beta=\omega n_{m} / c$. Above the light line, also in absence of losses, the propagation constant becomes complex. The dispersion curve we find is very different from previous studies, as we are solving dispersion relation for complex $\beta$ at a fixed real $\omega$. For example, considering the longitudinal mode $L$, we can see that above the light line the real part of the propagation constant describes approximately an arc of circumference, that intersects light line in two points (normalized frequency around 0.17 and 0.13 ). In the analogous case studied in Ref. [9], the real part of dispersion curve describes approximately a parabola with vertex centered in $\beta=0$, implying only an intersection with light line. These differences are explained by the fact that above the light line the losses are very high and modifies also the real part of propagation constant. In view of these aspects we can assert that above the light line, calculating the dispersion curve by fixing real wavevector leads to totally wrong results.

The complex band diagram shows a second transverse mode $T_{2}$ with negative group 
velocity that has complex propagation constant (i.e. decays during propagation) even in absence of losses also below the light line. At the border of the first Brillouin zone $(\beta d /(2 \pi)=0.5)$ the propagation constant has a fixed real part $(\operatorname{Re}[\beta d /(2 \pi)]=0.5)$ and varying imaginary part. This mode has been overlooked in previous studies, as it is not possible to find by fixing a real wavevector. Physically it corresponds to an evanescent mode that has a complex propagation constant and decays oscillating during propagation. It indicates the presence of a frequency stop-band due to the periodic nature of the waveguide.

When losses are considered the dispersion curves change dramatically (circles and crosses in the figures). A first general feature is that zero group velocity is not allowed and the curves $\operatorname{Re}[\beta(\omega)]$ bend in order to avoid points where $\partial \operatorname{Re}[\beta] / \partial \omega=0$. For example the longitudinal mode displays zero group velocity at the edge of the Brillouin zone in absence of losses. When losses are included the real part of propagation constant turns backward at around $\operatorname{Re}[\beta] d /(2 \pi) \approx 0.48$.

The real part of the dispersion curve for transverse mode $T_{1}$ does not depart to much from the lossless curve for normalized frequencies below 0.165 and the imaginary part is very low. For higher frequencies, up to 0.17 , the losses increases and the the real part of $\beta$ is smaller than the lossless case and reaches the maximum value of 0.35 for a normalized frequency approximatively equal to 0.17 , that corresponds to null group velocity for lossless particles. Increasing further the frequency causes a fold of the curve that follows now the (lossless) $T_{2}$ mode and intersects again with the light 
line at the point $(\omega d /(2 \pi c), k d /(2 \pi))=(0.26,0.1735)$. This behavior is reminiscent of the surface plasmon mode at the straight interface between a lossy metal and a dielectric [23].

As far as mode $T_{2}$ is considered, the effect of losses is to smoothen the real part of the dispersion curve eliminating the edge at the junction of the two branches of the lossless $T_{2}$ curve $($ at $(\omega d /(2 \pi c), k d /(2 \pi))=(0.17,0.5))$. The influence of material losses in this case is quite small, since the imaginary part of propagation constant is high also in the lossless case.

This discussion shows that when a real metal is considered, the losses are so high that the effects on the dispersion curves cannot be treated by first order perturbation, as it is evident from the big influence of absorption also in the real part of propagation constant.

\section{Finite element simulations}

In order to asses the validity and to check the accuracy of the model described in the previous sections, we solved Maxwell's equations with a finite element method. The usual way to calculate the Bloch modes of a periodic structure is to fix the wavevector and to find the frequency by solving a linear eigenvalue problem. Since the metal is strongly dispersive, this entails an iterative cycle that can require several iterations, it is possible to evaluate only a mode at once and the iterations can even not converge. In order to avoid these complications we reformulate the problem into 
a quadratic eigenvalue problem for the wavevector at a fixed frequency. While the quadratic eigenvalue equation is nonlinear, it is a more tractable problem than the general nonlinear case above, and can yield solutions more efficiently. In addition, this formulation inherently yields bands of purely imaginary- and complex-wavevector Bloch modes, which may be particularly hard to obtain with nonlinear search routines. A similar formulation was exploited previously for the calculation of complex Bloch bands of a 2D plasmonic crystal [24].

Starting from Maxwell's equations in frequency domain, we eliminate the electric field and write the vector wave equation for the magnetic field in the domain $\Omega \in \mathbf{R}^{3}$, representing the unit cell of the structure:

$$
\nabla \times \varepsilon^{-1} \nabla \times \tilde{H}=\left(\frac{\omega}{c}\right)^{2} \tilde{H} \quad \text { in } \Omega
$$

The unit cell $\Omega$ is a volume of size $L_{x}, L_{y}, L_{z}$, where $L_{x}=d$ is fixed by the periodicity of the chain along $x$, and $L_{y}, L_{z}$ are arbitrary. A good choice of these two lengths (for numerical purpose) implies that the mode amplitude at the cell boundary must be decayed to a negligible value.

Due to the periodicity of the dielectric constant $\varepsilon$, we can write $\tilde{H}=H e^{i \mathbf{k} \cdot \mathbf{x}}$, where $\mathbf{k}=\left(k_{x}, k_{y}, k_{z}\right)$ is the wavevector and $H$ is periodic, with basic cell the domain $\Omega$. By inserting this ansatz in Eq.(20) and following the Galerkin procedure [25], we can 
write the variational formulation of the problem:

find $\omega \in \mathbf{R}$ such that $\exists 0 \not \equiv H \in V$ :

$$
\begin{aligned}
& \int_{\Omega} \varepsilon^{-1}(\nabla+i \mathbf{k}) \times H \cdot \overline{(\nabla+i \mathbf{k}) \times \phi}=\left(\frac{\omega}{c}\right)^{2} \int_{\Omega} H \cdot \bar{\phi} \\
& \forall \phi \in V,
\end{aligned}
$$

where $\phi: \Omega \rightarrow \mathbf{R}^{3}$ are test functions chosen from an appropriate functional space $V$ (details can be found in $[26,27]$ and references therein). After some algebra and by exploiting the vector identities $(a \times b) \cdot c=a \cdot(b \times c)$ and $(a \times b) \cdot(a \times c)=$ $|a|^{2}(b \cdot c)-(a \cdot b)(a \cdot c)$, we can rewrite the variational formulation of (21) for the wavenumber:

find $\beta \in \mathbf{C}$ such that $\exists 0 \not \equiv H \in V$ :

$$
\begin{aligned}
& \int_{\Omega} \varepsilon^{-1}(\nabla \times H) \cdot(\nabla \times \phi)-\left(\frac{\omega}{c}\right)^{2} \int_{\Omega} H \cdot \phi= \\
& -i \beta\left\{\int_{\Omega} \varepsilon^{-1} \hat{k} \cdot(H \times \nabla \times \phi)-\int_{\Omega} \varepsilon^{-1} \hat{k} \cdot(\phi \times \nabla \times H)\right\} \\
& -\beta^{2} \int_{\Omega} \varepsilon^{-1}\{H \cdot \phi-(\hat{k} \cdot H)(\hat{k} \cdot \phi)\}, \quad \forall \phi \in V,
\end{aligned}
$$

where $\beta=\sqrt{k_{x}^{2}+k_{y}^{2}+k_{z}^{2}}$ is the modulus of wavevector and $\hat{k}$ is its unit vector.

This integro-differential equation may be transformed into matrix format by following the usual finite element method discretization [25]: the domain $\Omega$ is divided into several tetrahedral subdomains (elements) in which locally supported expansion functions are defined; $H$ is expanded in terms of such functions within each element; and $\phi$ is taken to be each one of the local expansion function inside each element. 
Then the following matrix eigenvalue equation in $\beta$ results:

$$
\left(\mathbf{A}-\frac{\omega^{2}}{c^{2}} \mathbf{B}\right) \mathbf{x}=i \beta(\mathbf{C}-\mathbf{D}) \mathbf{x}+\beta^{2} \mathbf{F} \mathbf{x}
$$

Here $\mathbf{x}$ is a vector containing the coefficients of the expansion of $H$, and matrices $\mathbf{A}$ to $\mathbf{F}$ may be individually related to each integral in Eq. (22) by inspection. Explicit expressions for the matrices in this case can be found in [25]. The eigenvalue equation may be solved at a fixed frequency $\omega$ (and thus fixed $\epsilon(\omega)$ ) and $\mathbf{k}$-vector direction. The eigenvalue itself is the $\mathbf{k}$-vector amplitude, $\beta$. The most common way of solving the quadratic eigenvalue problem is by linearization, which results in a (linear) system twice the original size. We implemented the formulation (22) with a commercial finite element software [28].

In the following we show the numerical results for the two low-loss modes $T_{1}$ and $L$.

Figure 3 shows the real and imaginary parts of the dispersion curve $\beta_{T_{1}}(\omega)$ calculated by solving dispersion relation (18) and by finite elements. We can see that the agreement between the two methods of solution is almost perfect, both for the real and imaginary part of wavevector: finite elements simulations confirm the band folding and the impossibility of reaching zero group velocity. In fact, at least for the positive slope branch of the dispersion curve, we can affirm that the losses are inversely proportional to the group velocity and fix a lower limit to attainable group velocity. Once reached this lower limit, the band folds back and the losses increase dramatically. By looking at the imaginary part of the mode wavevector, we can es- 
timate a useful propagation bandwidth from normalized frequencies in the interval $(0.155,0165)$, corresponding to a wavelength range of $(450 \mathrm{~nm}, 480 \mathrm{~nm})$. The insets show three different slices $(x=0, z=0, y=0$ planes) of the norm of the magnetic field for the transverse mode at $\omega d /(2 \pi c)=0.16$. The doughnut shape visible in the $x-y$ plane implies an electric dipole mode sitting in the $y-z$ plane, transverse to the direction of alignment of the spheres ( $x$ axis), as expected.

Figure 4 shows the real and imaginary parts of the dispersion curve $\beta_{L}(\omega)$ calculated by solving dispersion relation (19) and by finite elements. In this case the finite element solution shows an appreciable deviation from the CDA model. For the real part we can see that the simulated propagation constant is always greater than the analytical one by a factor $\approx 10 \%$. Imaginary part agrees better for normalized frequencies up to 0.186 , and then begins to deviate consistently. These effects can be ascribed to the fact that for high frequencies we cannot neglect the influence of multi-poles modes. The insets show three different slices $(x=0, z=0, y=0$ planes $)$ of the norm of the magnetic field for the transverse mode at $\omega d /(2 \pi c)=0.183$. The doughnut shape visible in the $y-z$ plane implies an electric dipole mode sitting along the direction of alignment of the spheres ( $x$ axis), as expected.

\section{Conclusions}

In this paper we derived the exact dispersion relation for nanoparticle chains by exploiting Mie scattering method. In a certain range of parameters only the first 
order spherical vector harmonics need to be considered, and simple expressions for longitudinal and transverse modes are obtained, that we showed to reduce to the usual one obtained by CDA (provided that the polarizability of the spheres is calculated by Mie scattering coefficients). We found a complex dispersion curve that strongly differs from previous studies: losses modify even the real part of the propagation constant and prevent the existence of resonator modes characterized by vanishing group velocity. The results of the Mie model agree very well with the exact Bloch modes dispersion calculated by finite element solution of Maxwell's equations. 


\section{References}

1. M. Quinten, A. Leitner, J.R. Krenn, and F. R. Aussenegg, "Electromagnetic energy transport via linear chains of silver nanoparticles," Opt. Lett. 23, 13311333 (1998).

2. M. L. Brongesma, J. W. Hartman, and H. A. Atwater, "Electromagnetic energy transfer and switching in nanoparticle chain arrays below the diffraction limit," Phys. Rev. B 62, R16356-R16359 (2000).

3. S. A. Maier, P. G. Kik, and Harry A. Atwater, "Optical pulse propagation in metal nanoparticle chain waveguides," Phys. Rev. B 67, 205402 (2003).

4. W. H. Weber and G. W. Ford, "Propagation of optical excitations by dipolar interactions in metal nanoparticle chains," Phys. Rev. B 70, 125429 (2004).

5. D. S. Citrin, "Coherent excitation transport in metal-nanoparticle chains," Nano Lett. 4, 1561-1565 (2004).

6. R. A. Shore and A.D. Yaghjian, "Travelling electromagnetic waves on linear periodic arrays of lossless spheres," Electron. Lett. 41, 578-580 (2005).

7. C. R. Simovsky, A. J. Viitanen, and S. A. Tretyakov, "Resonator mode in chains of silver nanoparticles and its possible application," Phys. Rev. E 72, 066606 $(2005)$.

8. D. S. Citrin, "Plasmon-polariton transport in metal-nanoparticle chains embedded in a gain medium", Opt. Lett. 31, 98-100 (2006). 
9. A. F. Koenderink and A. Polman, "Complex response and polariton-like dispersion splitting in periodic metal nanoparticle chains," Phys. Rev. B 74, 033402 (2006).

10. A. Alú and N. Engheta, "Theory of linear chains of metamaterial/plasmonic nanoparticles as a subdiffraction optical nanotrasmission lines," Phys. Rev. B 74, $205436(2006)$.

11. V. A. Markel and A.K. Sarychev, "Propagtion of surface plasmons in ordered and disordered chains of nanoparticles," Phys. Rev. B 75, 085426 (2007).

12. A. A. Govyadinov and V. A. Markel, "From slow to superluminal propagation: Dispersive properties of surface plasmon polaritons in linear chains of metallic nanospheroids," Phys. Rev. B 78, 035403 (2008).

13. J. N. Anker, W. P. Hall, O. Lyanders, N. C. Shan, J. Zhao, and R. P. Van Duyne, "Biosensing with plasmonic nanosensors," Nature Materials 7, 442-453 (2008).

14. N. Fang, H. Lee, C. Sun, and X. Zhang, "Sub-diffraction-limited optical imaging with a silver superlens," Science 308, 534-537 (2005).

15. E. Ozbay, "Plasmonics: Merging photonics and electronics at nanoscale dimensions," Science 311, 189-193 (2006).

16. J. M. Gerardy and M. Ausloos, "Absorption spectrum of spheres from the general solution of Maxwell's equations. II. Optical properties of aggregated metal spheres," Phys. Rev. B 25, 4204-4229 (1982). 
17. J. Stratton, Electromagnetic theory, McGraw-Hill (1941).

18. C. F. Bohren and D. R. Huffman, Absorption and Scattering of Light by Small Particles, Wiley (1983).

19. Y-l. Xu , "Fast evaluation of the Gaunt coefficients," Math. Comput. 65, 1601$1612(1996)$.

20. L. Lewin, Structural properties of Polylogarithms, American Mathematical Society (1991).

21. W. T. Doyle, "Optical propteries of a suspension of metal spheres," Phys. Rev. B 14, 9852-9858 (1989).

22. P. B. Johnson and R. W. Christy, "Optical constants of noble metals," Phys. Rev. B, vol. 6, 4370-4379 (1972).

23. S. A. Maier, Plasmonics: Fundementals and Applications, Springer (2007), p. 29.

24. M. Davanco, Y. Uhzhumov, and G. Shvetz, "The complex Bloch bands of a 2D plasmonic crystal displaying isotropic negative refraction," Opt. Express 15, 9861$9691(2007)$

25. J. Jin, The finite element method in electromagnetics, Wiley (2002).

26. J.-C. Ndlec, "Mixed finite elements in $R^{3}$," Numer. Math. 35, 315-341 (1980).

27. D. Boffi, M. Conforti, and L. Gastaldi, "Modified edge finite elements for photonic crystals," Numer. Math. 105, 249-266 (2006).

28. http://www.comsol.com 


\section{Figure Captions}

Figure1: Real part of the roots $\beta(\omega)$ of the dispersion relations (18-19). Dotted curves, lossless metal; circles and crosses, lossy metal.

Figure 2: Imaginary part of the roots $\beta(\omega)$ of the dispersion relations (18-19). Dotted curves, lossless metal; circles and crosses, lossy metal. The horizontal line $\omega d /(2 \pi c) \approx 0.169$ indicates the zero group velocity of the transverse mode $T_{1}$ in the lossless case.

Figure 3: Transverse mode $T_{1}$. Real and imaginary part of the roots $\beta(\omega)$ of the dispersion relations (18) (solid line) and results of finite element simulation (circles). Insets show three slices $(x=0, z=0, y=0$ planes) of the norm of the magnetic field for the transverse mode at $\omega d /(2 \pi c)=0.16$.

Figure 4: Longitudinal mode $L$. Real and imaginary part of the roots $\beta(\omega)$ of the dispersion relations (19) (solid line) and results of finite element simulation (circles). Insets show three slices $(x=0, z=0, y=0$ planes $)$ of the norm of the magnetic field for the transverse mode at $\omega d /(2 \pi c)=0.183$. 


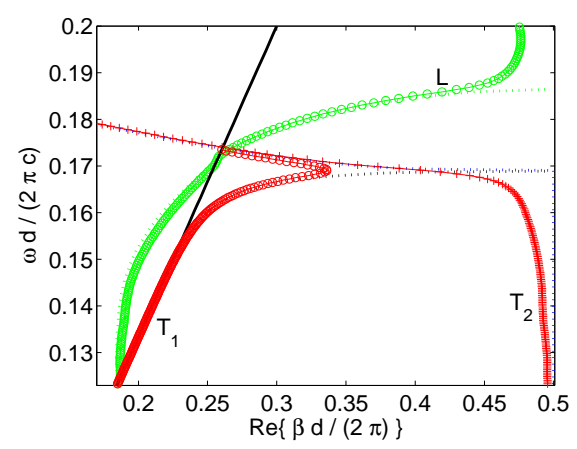

Fig. 1. Real part of the roots $\beta(\omega)$ of the dispersion relations (18-19). Dotted curves, lossless metal; circles and crosses, lossy metal. 


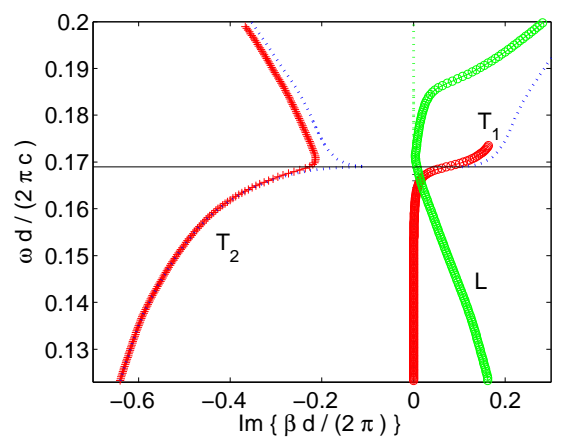

Fig. 2. Imaginary part of the roots $\beta(\omega)$ of the dispersion relations (18-19). Dotted curves, lossless metal; circles and crosses, lossy metal. The horizontal line $\omega d /(2 \pi c) \approx 0.169$ indicates the zero group velocity of the transverse mode $T_{1}$ in the lossless case. 

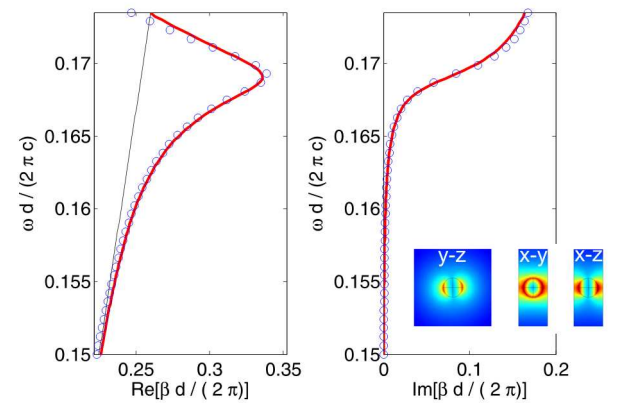

Fig. 3. Transverse mode $T_{1}$. Real and imaginary part of the roots $\beta(\omega)$ of the dispersion relations (18) (solid line) and results of finite element simulation (circles). Insets show three slices $(x=0, z=0, y=0$ planes) of the norm of the magnetic field for the transverse mode at $\omega d /(2 \pi c)=0.16$. 

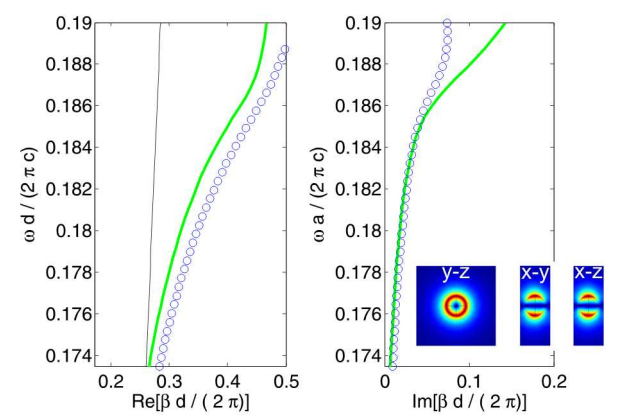

Fig. 4. Longitudinal mode $L$. Real and imaginary part of the roots $\beta(\omega)$ of the dispersion relations (19) (solid line) and results of finite element simulation (circles). Insets show three slices $(x=0, z=0, y=0$ planes) of the norm of the magnetic field for the transverse mode at $\omega d /(2 \pi c)=0.183$. 